\title{
IRiP
}

IRiP (Indonesian Review of Physics) is a journal of physics and physics education research. It is compiled with a commitment to enhance quality of the research. Indonesian Review of Physics (IRiP) aims to provide a forum for researchers, lecturers, teacher and student on the topics related to physics and physics education. The journal was first published in 2018 and regularly published twice per year in June and December.

\author{
Editor in Chief \\ Fatin Aliah Phang \\ Scopus ID: 57192376607 \\ Universiti Teknologi Malaysia \\ Johor Bahru, Malaysia \\ email: p-fatin@utm.my
}

\author{
Managing Editor \\ Dwi Sulisworo \\ Scopus ID:55317003800 \\ Universitas Ahmad Dahlan \\ Yogyakarta, Indonesia \\ email: dwi.sulisworo@uad.ac.id
}

\section{Advisory International Editorial Boards}

\author{
Akihiko Tomita \\ Scopus ID: 7201880070 \\ Wakayama University \\ Japan \\ atomita@center.wakayama-u.ac.jp
}

\author{
Muhammad Abdul Hadi \\ Bunyamin \\ Scopus ID: 57195100817 \\ Universiti Teknologi Malaysia \\ Malaysia \\ mabhadi@utm.my
}

Yudhiakto Pramudya

Scopus ID : 57192652457

Universitas Ahmad Dahlan Indonesia

yudhiakto.pramudya@pfis.uad.ac.id

\author{
Hassan K. Juwhari \\ Scopus ID: 6503859673 \\ University of Jordan \\ Jordan \\ juwhari@ju.edu.jo
}

\author{
Budhy Kurniawan \\ Scopus ID: 6602596727 \\ Universitas Indonesia \\ Indonesia \\ bkuru07@gmail.com
}

Noorali T Jiwaji
Scopus ID: 57190731624
Open University of Tanzania,
United Republic of Tanzania
noorali.jiwaj@out.ac.tz

Noorali T Jiwaji

pen University of Tanzania,

noorali.jiwaj@out.ac.tz

\author{
Rungchatchadaporn Vehachart \\ Scopus ID: 6992533300 \\ Thaksin University \\ Thailand \\ vahachart@hotmail.com
}

Moh Toifur
Scopus ID: 570242327000
Universitas Ahmad Dahlan
Indonesia
mtoifur1807@gmail.com
Johny Setiawan
Scopus ID: 8680503300
UNIMATRIX Verlag- Berlin,
Germany
setiawan@uni-matrix.de

\section{Editorial Asistant}

\section{Rizky Merian Muspa}

Universitas Ahmad Dahlan

Indonesia
Efi Kurniasari

Universitas Ahmad Dahlan

Indonesia

\section{Journal Manager}

Moh Irma Sukarelawan

Universitas Ahmad Dahlan

Indonesia

Publisher address:

Universitas Ahmad Dahlan (UAD)

Kampus 2 Universitas Ahmad Dahlan

Jalan Pramuka No. 42, Pandeyan, Umbulharjo, Yogyakarta - 55161

Telp. +62 274 563515, ext. 4902; Fax. +62 274564604

Email: irip@mpfis.uad.ac.id 


\section{IRiP}

(INDONESIAN REVIEW of PHYSICS)

Vol. 3 No. 1, June 2020

Regular Papers:

Effect of the Magnetic Force on Ferrite Pendulum Oscillation Parameters: Parametric Analysis on Ferrite Pendulum

Rusli Adi, Moh Toifur, Ishafit Ishafit, Rosly Jaafar

Measuring the Apparent Magnitude of Planet Mars on August 1st and October 2nd, 2018 at the Falak Observatory at Muhammadiyah University of North Sumatera Muhammad Hidayat, Arwin Juli Rakhmadi, Abu Yazid Raisal

Development of Interactive Physics E-Module Using the SETS (Science, Environment, Technology, Society) Approach to Improve Science Literacy Dimension of Content and Process Dimensions in Fluid Dynamics Material

Eka Syafutri, Widodo Widodo, Yudhiakto Pramudya

Analysis of Students' Understanding of Concepts on Momentum and Impulse Material Using Research-Based Learning (RBL) Models

Nanda Safarati, Rahma Rahma

Effect of Deposition Voltage on Layer Thickness, Microstructure, $\mathrm{Cu} / \mathrm{Ni}$ Sheet Resistivity of Deposition Results by Magnetic Field Electroplating Assisted Technique Willi Anindita Wijanarka, Moh Toifur

Implementation Cooperative Learning Type STAD Assisted Edmodo in Improving Students' Critical Thinking Skills

Muhamad Epi Rusdin, Widodo Widodo, Guntur Maruto

Published by

Universitas Ahmad Dahlan

\begin{tabular}{|c|c|c|c|c|c|}
\hline IRiP & Vol.3 & No.1 & pp. 1-34 & $\begin{array}{c}\text { Yogyakarta, } \\
\text { June 2020 }\end{array}$ & $\begin{array}{c}\text { p-ISSN 2621-3761 } \\
\text { e-ISSN 2621-2889 }\end{array}$ \\
\hline
\end{tabular}




\section{Table of Contents}

\section{Regular Papers:}

Effect of the Magnetic Force on Ferrite Pendulum Oscillation Parameters:

Parametric Analysis on Ferrite Pendulum

Rusli Adi ${ }^{1}$, Moh Toifur ${ }^{1}$, Ishafit Ishafit ${ }^{1}$, Rosly Jaafar ${ }^{2}$

1Universitas Ahmad Dahlan, Yogyakarta, Indonesia

${ }^{2}$ Universiti Pendidikan Sultan Idris, Kuala Lumpur, Malaysia

Measuring the Apparent Magnitude of Planet Mars on August $1^{\text {st }}$ and October $2^{\text {nd }}, 2018$ at the Falak Observatory at Muhammadiyah University of North Sumatera

Muhammad Hidayat, Arwin Juli Rakhmadi, Abu Yazid Raisal

Universitas Muhammadiyah Sumatera Utara, Sumatra Utara, Indonesia

Development of Interactive Physics E-Module Using the SETS (Science,

Environment, Technology, Society) Approach to Improve Science Literacy Dimension of Content and Process Dimensions in Fluid Dynamics Material

Eka Syafutri, Widodo Widodo, Yudhiakto Pramudya

Universitas Ahmad Dahlan, Yogyakarta, Indonesia

Analysis of Students' Understanding of Concepts on Momentum and Impulse Material Using Research-Based Learning (RBL) Models

Nanda Safarati, Rahma Rahma

Universitas Almuslim, Aceh, Indonesia

Effect of Deposition Voltage on Layer Thickness, Microstructure, Cu/Ni Sheet Resistivity of Deposition Results by Magnetic Field Electroplating Assisted Technique

Willi Anindita Wijanarka1, Moh Toifur²

${ }^{1}$ SMP Muhammadiyah 1 Mlati, Yogyakarta, Indonesia

${ }^{2}$ Universitas Ahmad Dahlan, Yogyakarta, Indonesia

Implementation Cooperative Learning Type STAD Assisted Edmodo in Improving Students' Critical Thinking Skills

Muhamad Epi Rusdin'1, Widodo Widodo ${ }^{2}$, Guntur Maruto ${ }^{3}$

IIKIP Muhammadiyah Maumere, Maumere, Indonesia

2Universitas Ahmad Dahlan, Yogyakarta, Indonesia

${ }^{3}$ Universitas Gajah Mada, Yogyakarta, Indonesia 